\title{
Restricted stimulus control in stimulus control shaping with a capuchin monkey
}

\author{
Ana Leda de Faria Brino ${ }^{1}$, Olavo de Faria Galvãoํㅜ, Romariz da Silva Barros ${ }^{1}$, Paulo Roney Kilpp \\ Goulart $^{1}$ and William J. McIlvane ${ }^{2}$ \\ 1- Universidade Federal do Pará, Belém, PA, Brazil
}

2- University of Massachussetts, Waltham, MA, USA

\begin{abstract}
Teaching the first instances of arbitrary matching-to-sample to nonhumans can prove difficult and time consuming. Stimulus control relations may develop that differ from those intended by the experimenter - even when stimulus control shaping procedures are used. We present, in this study, efforts to identify sources of shaping program failure with a capuchin monkey. Procedures began with a baseline of identity matching. During subsequent shaping trials, compound comparison stimuli had two components - one identical to and another different from the sample. The identical component was eliminated gradually by removing portions across trials (i.e., subtracting stimulus elements). The monkey performed accurately throughout shaping. At a late stage in the program, probe tests were conducted: (1) arbitrary matching trials that had all elements of the identical comparison removed and (2) other trials that included residual elements. During the test, the monkey performed at low levels on the former trials and higher levels on the latter. These results suggested that higher accuracy was due merely to continued control by the residual elements: the target arbitrary matching relations had not been learned. Thus, it appears that procedures that gradually transform identity matching baselines into arbitrary matching can fail by inadvertently shaping restricted control by residual elements. Subsequent probes at the end of the shaping series showed a successful transfer of stimulus control from identity to arbitrary matching after further programming steps apparently overcame the restricted stimulus control. Keywords: matching-to-sample, stimulus control shaping; restricted stimulus control, Cebus cf. apella.
\end{abstract}

Received 28 February 2012; received in revised form 1 June 2012; accepted 8 June 2012. Available online 29 June 2012.

\section{Introduction}

Stimulus control shaping procedures (cf. Mcllvane \& Dube, 1992) may be useful for establishing simple and conditional discrimination performances when mere differential reinforcement (trial-and-error) procedures prove ineffective or inefficient (e.g., Sidman \& Stoddard, 1967; Schilmoeller, Schilmoeller, Etzel, \& LeBlanc, 1979; Touchette \& Howard, 1984). Such procedures begin by establishing a baseline with relations that are already within the organism's repertoire or can be acquired easily. Thereafter, programmed changes are made in the stimuli from the original baseline, transforming them - typically gradually - into new stimuli thus establishing new stimulus control relations. Via this method, new relations may be established with few or no errors (e.g., Sidman \& Stoddard, 1967).

Ana Leda de Faria Brino, Olavo de Faria Galvão, Romariz da Silva Barros, Paulo Roney Kilpp Goulart, Experimental School for Primates, Nucleus of Theory and Research on Behavior, Federal University of Pará, Belém, Brazil; William J. McIlvane, E. K. Shriver Center, University of Massachusetts Medical School, Waltham, Massachusetts, U.S.A. Correspondence regarding this article should be directed to: Ana Leda de Faria Brino, Núcleo de Teoria e Pesquisa do Comportamento, Universidade Federal do Pará, Campus Professor José da Silveira Netto, Rua Augusto Correa, no 1, CEP: 66.075-110, Guamá, Belém, PA, Brazil. Phone: +55 9132018475. E-mail: abrino@gmail.com
One such procedure presented by Zygmont, Lazar, Dube, and Mcllvane (1992) has been an alternative for teaching humans with developmental limitations who show protracted failures to learn arbitrary matching-tosample (ArbMTS) performances via simple differential reinforcement. Termed sample stimulus control shaping, their procedure began with a well-established identity matching-to-sample (IDMTS) baseline. During shaping, sample stimuli were made increasingly less similar to the comparisons. At the end of shaping, samples and corresponding comparisons did not resemble each other; thus, an ArbMTS baseline was established.

Brino and colleagues (2010) systematically replicated these procedures with two capuchin monkeys (Cebus cf. apella). Their first experiment used the procedures of Zygmont and colleagues (1992) and obtained similar data. Their second varied the procedure by altering comparisons rather than samples. In 15 steps, identical elements of the comparisons were gradually removed, substituting instead horizontal bands of elements of the new comparisons. During the band removal/substitution procedure, the monkey maintained fairly high accuracy. At late stages of shaping, however, accuracy fell to low levels. Results of probe tests suggested that accurate matching depended upon the presence of small residual elements of comparison stimuli that "matched" identical elements in the sample stimuli. Arbitrary matching relations were not 
established. Maintained stimulus control was apparently restricted to the situation in which residual elements were present - analogous to the "stimulus overselectivity" findings reported in children with autism and other developmental limitations (e.g., Lovaas, Schreibman, Koegel, \& Rehm, 1971; Litrownik, Mc-Innis, WetzelPritchard, \& Filipelli, 1978).

The study reported here systematically replicated the second study of Brino and colleagues (2010), investigating an alternative to band removal/substitution. Comparison stimuli from the IDMTS baseline were superimposed on comparison stimuli that were to constitute the target arbitrary matching relations, thus creating compound comparison stimuli. During shaping, identical elements of the compound were gradually eliminated by progressive deletion from their edges across trials. When all elements were eliminated, the monkey was only able to continue to match accurately if the target arbitrary sample-comparison relations had been learned. This study also included stimulus control probes to assess whether program failures may be attributable to restricted stimulus control by identical elements as suggested by the findings of Brino and colleagues (2010).

\section{Methods}

\section{Subject}

Raul, an adult male capuchin, participated. $\mathrm{He}$ participated in previous studies of simple discrimination, discrimination reversal, and identity matching-to-sample (Galvão et al., 2005; Brino, Galvão, \& Barros, 2009). Morning sessions were run from Monday to Friday each week. The animal's regular feeding occurred daily at $3 \mathrm{pm}$.

Raul lived with other three capuchins in an external cage $(2.5 \times 2.5 \times 2.5 \mathrm{~m})$ adjacent to the laboratory. Four small auxiliary cages $(0.5 \times 0.5 \times 0.5 \mathrm{~m})$ attached to the main cage were used to separate the animals while they were fed. A fifth small cage, featuring sliding doors on inside and outside walls, facilitated transfer into a portable cage that was used to transport the animal to the experimental chamber for sessions.

\section{Apparatus}

The chamber $(0.80 \times 0.80 \times 0.70 \mathrm{~m})$ was mounted in an aluminum superstructure within a larger cubicle $(2.5 \times 1.9 \times 2.9 \mathrm{~m})$. The floor, ceiling, and left wall of the chamber were made of steel screen. The right and front walls were metal plate. One could access the chamber through a hinged door $(0.35 \times 0.20 \mathrm{~m})$ on the left wall. The chamber was equipped with a touchscreen color monitor. Raul could respond to its screen by reaching through a rectangular opening $(0.26 \times 0.20 \mathrm{~m})$ on the front wall. Centered $24 \mathrm{~cm}$ below the opening was a receptacle for delivering $190 \mathrm{mg}$ Noyes banana pellets via a hose connected to a Med Associates dispenser. All stimulus presentation and response recording was managed by a microcomputer (AMD K6 150).

\section{Stimuli}

We used two sets of shapes (A [black] and B [gray]), both presented on square white backgrounds. Each shape measured $2.43 \times 2.43 \mathrm{~cm}$. Sample and comparison stimuli used in the shaping procedure are shown in Figure 1.

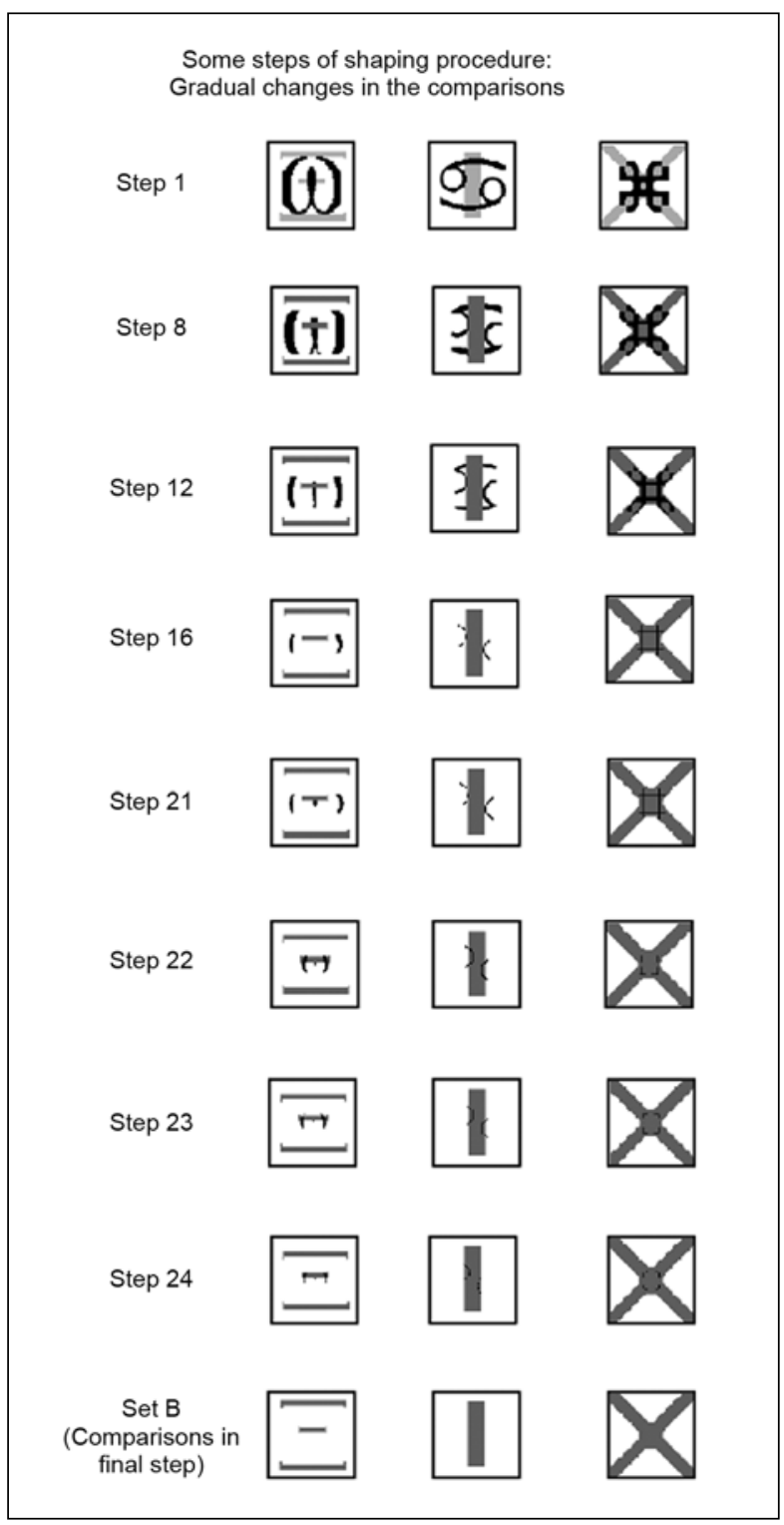

Figure 1. Selected steps of the stimulus control shaping procedure. Stimuli from Set A were superimposed on the stimuli from Set B in the comparisons.

\section{General procedure}

A 0-delay, 3-choice MTS procedure was used. Each trial began with presentation of a sample stimulus in any of nine positions of a $3 \times 3$ matrix on the computer screen. A touch to the sample was followed by sample removal and presentation of three comparison stimuli. Comparison locations also varied unsystematically across the nine positions and were counterbalanced across trials. A touch to the comparison defined as $\mathrm{S}+$ (to be matched to the sample) was followed immediately by a food pellet and a 4-s intertrial interval (ITI). A touch 
to any S- was followed by only the ITI. Sessions ended after a pre-established number of trials (typically 48) or after $25 \mathrm{~min}$, whichever happened first.

\section{Training}

Initially, Raul was given IDMTS training with the stimuli from Sets A and B, alternating A-A and B-B trials in the same session. After reaching a criterion of $90 \%$ correct responses in two consecutive sessions, a stimulus control shaping procedure was implemented to try to establish arbitrary relations (A1-B1, A2-B2, and A3-B3). Selected steps of the program are shown in Figure 1. Sample stimuli were always from Set A. Comparison stimuli were composed of Set A stimuli superimposed on Set B stimuli.

The shaping procedure was a variant of the "superimposition plus fading" method that has its roots in early work by Terrace (1963). In that study, vertical and horizontal lines were initially superimposed on an established red vs. green simple discrimination presented in the successive discrimination format. Over shaping trials, the colors were gradually faded out with the goal of transferring stimulus control to some aspect of the vertical vs. horizontal stimulus difference. The method achieved errorless transfer in pigeons. The present procedures, in contrast, were conducted in the context of conditional discrimination (IDMTS) involving simultaneously discriminated identical A comparison stimuli, elements of which were gradually erased to reveal elements of the dissimilar B comparison stimuli. Typically, element erasures occurred with all three members of Set A on each program step, but that procedure was sometimes varied in an effort to facilitate acquisition.

IDMTS and ArbMTS criteria were 90\% correct responses with errors proportionally distributed among relations. Sessions featuring a "blank comparison" (Mcllvane et al., 1987) or "mask" procedure (MK) were conducted after criterion was met at each program step with the objective of verifying both S- independent sample-S+ select relations and $\mathrm{S}+$ independent sample-Sreject matching relations. In the mask procedure, one comparison was replaced by a blank window (the white background with no form on it). If the mask replaced an $\mathrm{S}+$ stimulus on a given trial, then it became $\mathrm{S}+$ because the other two comparison stimuli had been defined as $\mathrm{S}-$ in relation to the sample. If the mask replaced an Sstimulus, however, then the mask also was an S- because one other comparison stimulus had been defined as $\mathrm{S}+$ in relation to the sample. By verifying independent sample-S+ and sample-S- relations, the mask procedure was intended to increase the likelihood that Raul would acquire true sample-comparison relations rather than merely responding to each trial display as its own independent stimulus configuration (McIlvane, in press).

\section{Tests for Restricted Stimulus Control}

Tests were run after Step 21 (Tests 1 and 2) and Step 24 (Test 3 and 4) of the shaping protocol. They evaluated which components of comparison stimuli were involved in the stimulus control of accurate matching at that step. One probe type (Tests 1 and 3) displayed Set A samples with the physically different Set B elements that were introduced during shaping (i.e., final performance arbitrary matching trials). Another probe type (Tests 2 and 4) displayed samples from Set A with comparisons containing residual identical elements that had not been changed during shaping. If stimulus control had transferred as intended, then accurate matching would occur on Tests 1 and 3. If not, then Raul could exhibit accurate matching only if he was able to match identical stimulus elements.

Each test session had 48 trials: 12 probe trials (four of each relation, A1B1, A2B2, and A3B3) were interspersed in 36 baseline trials (12 of each relation at the corresponding step in shaping). Figures 2 and 3 show the stimuli that were presented on the probe trials.

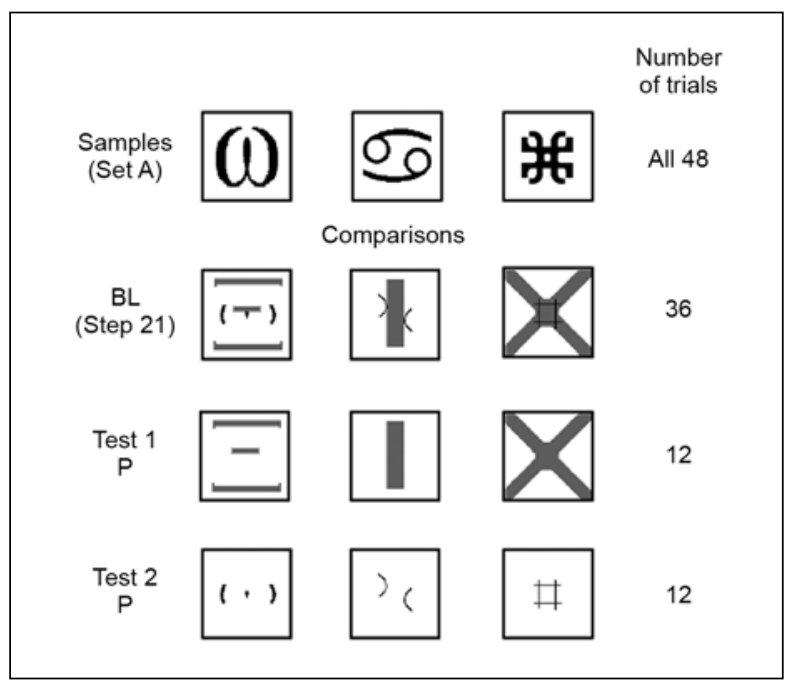

Figure 2. Sample (Set A) and comparisons presented in baseline (BL) and probe trials $(\mathrm{P})$ of Tests 1 and 2 to evaluate stimulus control by the components of the comparisons from Step 21.

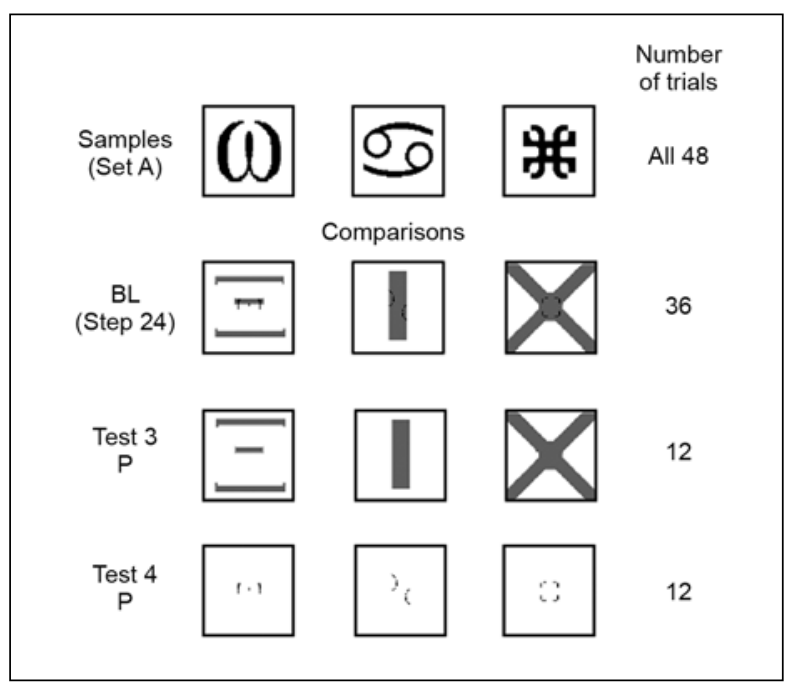

Figure 3. Sample (Set A) and comparisons presented in baseline (BL) and probe trials $(\mathrm{P})$ of Tests 3 and 4 to evaluate stimulus control by the components of the comparisons from Step 24. 


\section{Results}

Figure 4 shows the average percentage of correct responses (top panel), standard deviation (middle panel), and number of sessions to criteria (bottom panel) at the steps of the shaping program prior to achieving criterion. These data show that the shaping protocol maintained step-to-step accuracy at reasonably high levels. Errors following stimulus changes were few and typically limited to one of the relations. Accuracy on baseline trials during subsequent probe sessions was also high (range: $91.7 \%$ to $100 \%$ ).

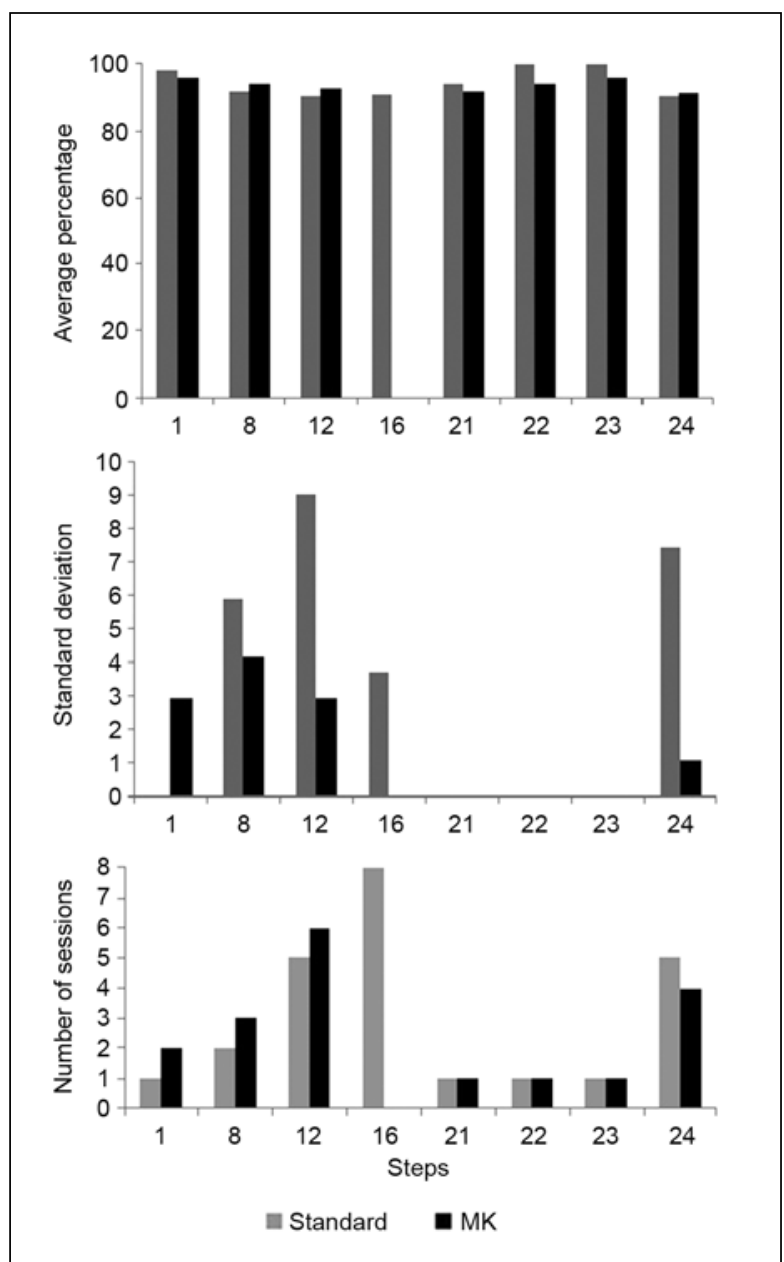

Figure 4. Average percentage of correct responses (top panel) and standard deviation (middle panel) for those steps of shaping procedure in which more than one session was necessary for reaching performance criteria. Number of standard and Mask ArbMTS sessions to criteria $(90 \%$ of correct responses and errors distributed among relations) for selected steps of the shaping procedure (bottom panel).

Tests 1 and 2. These results are shown in Figure 5. Accuracy on Test 1 was only $42 \%$ (comparable to nonconditional sample-independent responding-33\% on a three-choice task, sometimes termed 'chance'). No individual Test 1 probe trial accuracy exceeded $50 \%$. These data indicated virtually complete failure of the intended transfer of stimulus control from identical to dissimilar elements at that point in the program.
In contrast, matching accuracy on Test $2(67 \%)$ was well above the level expected if comparison responding was merely sample-independent responding. Moreover, combined accuracy on two of three Test 2 probe trials $(88 \%)$ approximated baseline trial accuracy levels, whereas the third was very low $(25 \%)$. We interpreted these results as (1) retained stimulus control by residual identical controlling elements on two probe trial types and (2) sample-independent responding on the third, due perhaps to removal of the controlling element(s)_possibly a compound stimulus involving the residual identical elements and elements of the dissimilar stimulus. In attempting to account for the latter, we noted that residual identical elements of the probe $\mathrm{A} 3 \mathrm{~B} 3$ relation were entirely within the boundaries of the dissimilar components during shaping, whereas the residual identical elements of the other two probe relations overlapped and exceeded the boundaries of the dissimilar components (see Figure 1, Steps 12-21).

In designing subsequent program Steps 22-24, we decided to place the residual identical elements for all three relations within the boundaries of the dissimilar elements. We reasoned that observation of the latter might be encouraged if Raul was simultaneously observing the former (see a discussion by Ray [1969] who discussed the concept of shared control in stimulus control transfer).

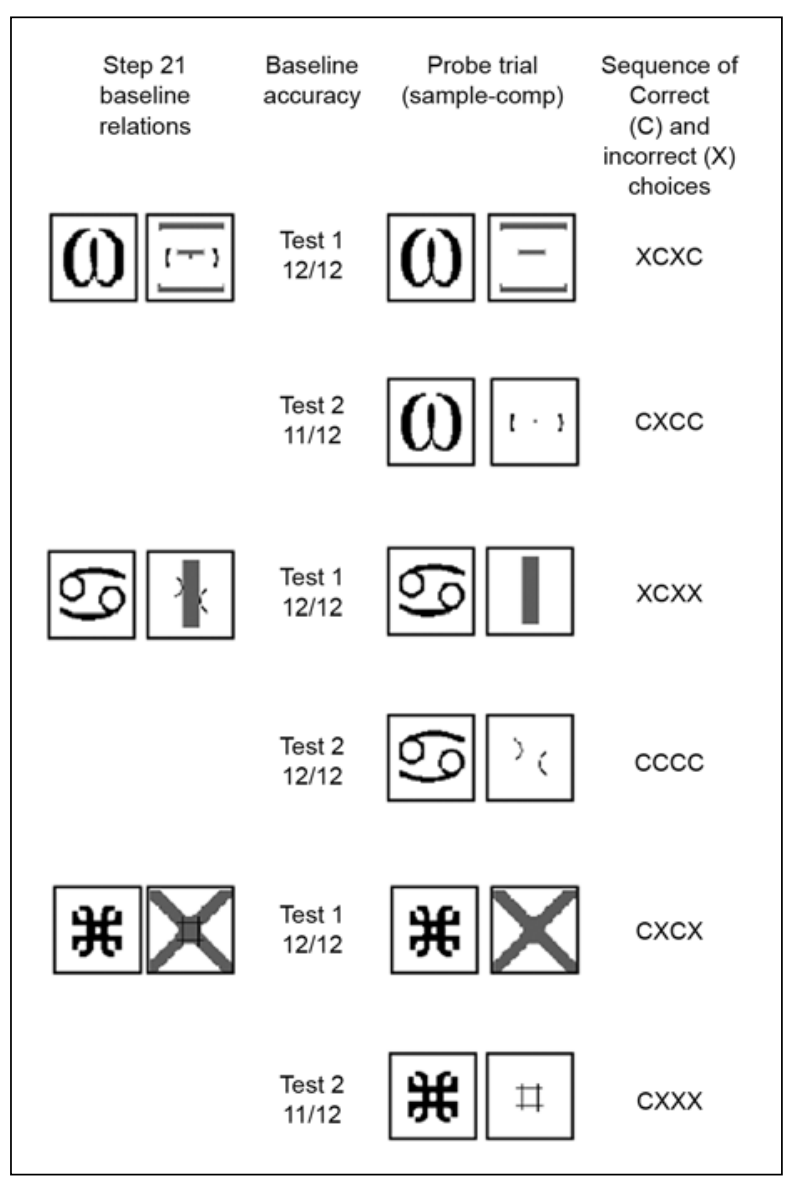

Figure 5. Results of Tests 1 and 2. For each relation in test session, we present the baseline accuracy (number of correct responses by the total number of trials), and the accuracy on probe trials (sequence of correct $[\mathrm{C}]$ and incorrect $[\mathrm{X}]$ choices). 
Tests 3 and 4. Conducted after Step 24 of the shaping protocol, these results are shown in Figure 6. Raul exhibited reasonably accurate matching on Test $3(88 \%)$ but no apparent conditional control by the samples on Test $4(25 \%)$. These results are consistent with (1) successful transfer of sample stimulus control from identical components to dissimilar components (i.e., arbitrary matching) and (2) no control by the now miniscule residual elements that had all but disappeared at the end of the shaping protocol.

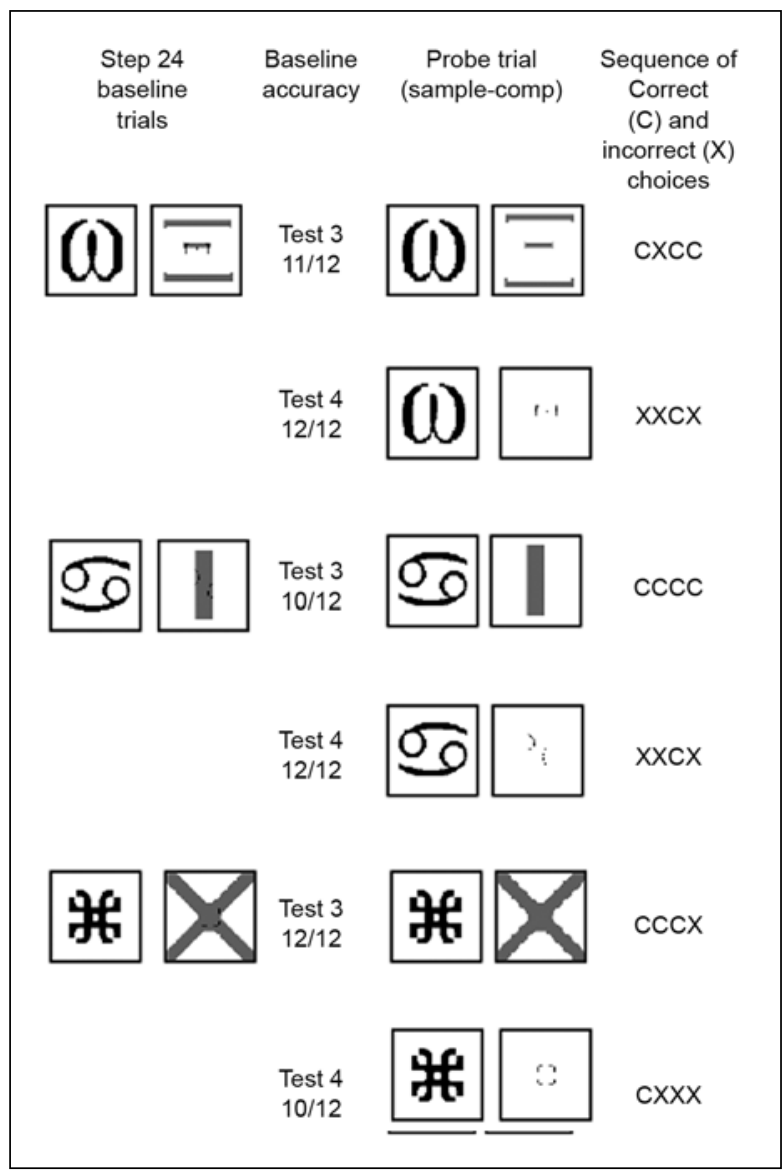

Figure 6. Results of Tests 3 and 4 . for each relation in the test session, we present the baseline accuracy (number of correct responses divided by the total number of trials), and the accuracy on probely trials (sequence of correct $[\mathrm{C}]$ and incorrect $[\mathrm{X}]$ choices).

\section{Discussion}

In the present study we investigated a stimulus control shaping procedure aimed at teaching arbitrary matching-to-sample beginning with an identity matching-to-sample baseline. When one employs such a procedure, the assumption is made that gradual manipulation of stimulus features will direct the subject's attending to those stimulus features that are relevant in arbitrary matching. Although this outcome occurred at the final steps of our shaping program, the findings of Tests 1 and 2 suggested that no such transfer occurred through Step 21. Rather, the shaping procedure apparently merely directed attending to the unchanged elements of the comparison compounds. Accuracy when the dissimilar arbitrary matching comparison stimuli were presented by themselves was at virtual chance-level at that point.

One interpretation of our findings is that the original controlling elements "blocked" the development of control by those elements that we wanted to gain control. However, we strongly prefer a conceptualization in terms of restricted stimulus control (or "overselective" attending) in the present case. Analyses in terms of blocking are unhelpful in explaining why transfer often occurs without errors (e.g., as in the Terrace [1963] study) in situations that seem to have the potential for blocking. Moreover, attributing our findings to blocking also does not explain why effective transfer (i.e., no blocking) occurred during the last stage of our stimulus control transfer procedure. In general, we believe that the controlling variables in effective stimulus control transfer will come to be understood increasingly in terms of securing and maintaining precise control of what and perhaps where the subject attends.

Our results reinforce the conclusion that, under certain conditions, stimulus control shaping procedures can produce controlling relations inconsistent with those intended by the experimenter. Inconsistent relations may be of the type illustrated in this study or other types such as unwanted control by stimulus compounds (McIlvane, in press). One contribution of the present study is the demonstration of a systematic procedure to reliably identify the actual ongoing stimulus control relations in nonverbal organisms. It contributes also in showing that changes in the comparison stimuli during shaping could produce restricted control in the same manner as had apparently occurred during sample stimulus control shaping with a different capuchin in the study by Brino et al. (2011). Unlike the results reported by Brino and colleagues (2011), reasonably high accuracy was observed on most steps of shaping training. Nevertheless, stimulus control by the arbitrary components was not consistently established until the final steps of the procedure.

McIlvane, Serna, Dube, and Stromer (2000) pointed out that high accuracy in a discrimination task can occur even when the established controlling relations actually differ from those intended by the experimenter. This was shown clearly by the fact that the stimulus control shaping procedure reported here resulted in high accuracy from Step 1 to Step 21. Although the simultaneous presentation of sample stimulus elements can result in the development of control by every stimulus element (Stromer \& McIlvane, 1993), Raul clearly attended only to restricted elements of the compound through Step 21. It was important, therefore, to find a procedure that could remediate the restricted stimulus control. Superimposing the stimulus features that we wanted to control within those that did control proved effective in this case, but we do not yet know whether this could be a generally effective stimulus control shaping strategy for capuchins.

To overcome or even bypass unwanted shaping outcomes, one needs to develop procedures to (1) make 
measurements that will allow one to infer the controlling relations that are developing and (2) re-program the shaping series if it appears that the program does not have its intended effects. Our observations buttress the case for designing procedures that measure controlling relations frequently during shaping (cf. Reynolds, 1961; Birkimer, 1969; Ray, 1969). For example, one could imagine procedures that use probe tests analogous to those used in this study toward the end of the shaping procedures after, e.g., every third step-with remedial procedures available for use whenever they appear to be needed. Such procedures would likely forestall the provision of lengthy histories of reinforcement for irrelevant, undesired controlling relations_ - histories that may render remedial procedures less efficient or even ineffective.

Going forward, it seems clear that development of restricted stimulus control can be an unwanted side effect of stimulus control shaping, suggesting the need to identify and explore ways to prevent this outcome. Among the possibilities are the following:

(1) A more expeditious procedure could be implemented with the participant advanced to the next step in shaping as soon as a reasonable criterion (e.g., six successive correct trials) is reached, rather than using overtraining as was employed in this study. Such a procedure may reduce the likelihood that controlling relations established earlier in the shaping program would persist to its later stages.

(2) Another approach could be to program stimulus changes in variable locations (e.g., different quadrants) of the identical components - such that no single stimulus feature would be an invariant predictor of reinforcement. This approach has had some success with human participants and should be investigated systematically with monkeys (Serna, 2004).

(3) It may help to equate the salience of stimuli to the extent possible. In our procedure, the identical component of the comparison and sample was always solid black and the different component was always a lighter gray. The darker quality may have encouraged maintenance of control by residual elements of the identical component.

(4) There may be benefits of investigating procedure alternatives to stimulus control shaping, perhaps using exclusion procedures in the context of an identity- or similarity-matching-to-sample baseline (Wilkinson, Rosenquist, \& McIlvane, 2009).

(5) Insertion of arbitrary relation probes (such as our Tests 2 and 4) occasionally throughout the shaping series has been used in preliminary studies in our laboratory. Results suggest that such procedures may facilitate acquisition of arbitrary matching relations (Brino, Assumpção, Campos, Galvão, \& McIlvane, 2010; cf. Fields, 1981).

As a final methodological note, we translated stimulus control shaping technology used to teach arbitrary matching relations originally with humans with intellectual disability (ID) to the task of teaching similar performances to monkeys. Whereas such shaping methodology may be effective in this application with both populations (Brino et al., 2010; Carr et al., 2000), we also found that the procedure sometimes has the unwanted outcome of shaping restricted stimulus control by unchanged elements in both. The fact that both humans with ID and monkeys apparently show virtually the same outcomes with similar procedures suggests that monkeys may be an especially valuable population for modeling certain learning processes in developmentally limited humans. An important question for future research is whether resolving learning challenges in discrimination training regimens with monkeys will lead directly to procedures for overcoming the same or similar challenges with such humans.

\section{Acknowledgements}

Support for this research was provided by the National Council for Scientific and Technologic Development (CNPq) to A.L.F. Brino (Doctoral Fellowship), O.F. Galvão (Research Productivity Fellowship), R.S. Barros (Research Productivity Fellowship), and P.R.K. Goulart (Master Fellowship). Additional support was provided by the Brazilian Ministry of Technology under the program of the National Institutes of Science and Technology on Behavior, Cognition, and Teaching (Program Announcement 15/2008, CNPq grant no. 573972/2008-7, FAPESP grant no. 2008/57705-8). W.J.M. was supported by Grants ES15464, MH90272 and HD04147 from the U.S. National Institutes of Health.

Living conditions, diet, veterinary care, and experimental protocols were approved by the Animal Research Ethics Committee of the UFPA (Universidade Federal do Pará) following local and international ethical guidelines. Protocols for the general care of the animals were also approved by IBAMA (Environment Brazilian Institute). We thank Klena Sarges for veterinarian assistance and Adilson Pastana for the excellent homecage care given to our monkeys.

We also thank José Iran A. dos Santos who developed the software package (TREL 2.1) that controlled the experimental procedures and William V. Dube for comments on an earlier version of the manuscript.

\section{References}

Augustson, K. G., \& Dougher, M. J. (1991). Teaching conditional discrimination to young children: Some methodogical successes and failures. Experimental Analysis of Human Behavior Bulletin, 9, 21-24. Retrieved from http://www.eahb.org/EAHB Bulletin_1991_V09_N02.pdf

Birkimer, J. C. (1969). Control of responding by the elements of a compound discriminative stimulus and by the elements as individual discriminative stimuli. Journal of the Experimental Analysis of Behavior, 12, 431-436. doi:10.1901/jeab.1969.12-431

Brino, A. L. F., Assumpção, A. P. B., Campos, R. S., Galvão, O. F., \& McIlvane, W. J. (2010). Cebus cf. apella exhibits rapid acquisition of complex stimulus relations and emergent performance by exclusion. Psychology \& Neuroscience, 3, 209-215. doi: 10.3922/j. psns.2010.2.010

Brino, A. L. F., Galvão, O. F., \& Barros, R. B. (2009). Successive identity matching to sample tests without reinforcement in Cebus apella. Ciências \& Cognição, 14, 2-11. Retrievedfrom http://www. cienciasecognicao.org 
Brino, A. L. F., Barros, R. S., Galvão, O. F., Garotti, M., Cruz, I. R. N., Santos, J. R...McIlvane, W. J. (2011). Sample stimulus control shaping and restricted stimulus control in capuchin monkeys: A methodological note. Journal of the Experimental Analysis of Behavior, 95, 387-398. doi:10.1901/jeab.2011.95-387

Carr, D., Wilkinson, K. M., Blackman, D., \& McIlvane, W. J. (2000). Equivalence classes in individuals with minimal verbal repertoires. Journal of the Experimental Analysis of Behavior, 74, 101-114. doi:10.1901/jeab.2000.74-101

Dixon, L. S. (1977). The nature of the control by spoken words over visual stimulus selection. Journal of the Experimental Analysis of Behavior, 27, 433-442. doi:10.1901/jeab.1977.27-433

Fields, L. (1981). Early and late introduction of probes and stimulus control acquisition in fading. Journal of the Experimental Analysis of Behavior, 36, 363-370.

Galvão, O. F., Barros, R. S., Santos, J. R., Brino, A. L. F., Brandão, S., Lavratti, C. M. ... McIlvane, W. J. (2005). Extent and limits of the matching concept in Cebus apella: A matter of experimental control? The Psychological Record, 55, 219-232. Retrieved from EBSCOhost

Litrownik, A. J., McInnis, E. T., Wetzel-Pritchard, A. M., \& Filipelli, D. L. (1978). Restricted stimulus control and inferred attentional deficits in autistic and retarded children. Journal of Abnormal Psychology, 87, 554-562. doi:10.1037/0021-843X.87.5.554

Lovaas, O. I., Koegel, R. L., \& Schreibman, L. (1979). Stimulus overselectivity in autism: A review of research. Psychological Bulletin, 86, 1236-1254. doi:10.1037/0033-2909.86.6.1236

Lovaas, O. I., \& Schreibman, L. (1971). Stimulus overselectivity of autistic children in a two stimulus situation. Behavior Research and Therapy, 9, 305-310. doi:10.1016/0005-7967(71)90042-8

Lovaas, O. I., Schreibman, L., Koegel, R. L., \& Rehm, R. (1971). Selective responding by autistic children to multiple sensory input. Journal of Abnormal Psychology, 77, 211-222. doi:10.1037/ h0031015

McIlvane, W. J. (in press). Simple and complex discrimination learning. Handbook of Behavior Analysis. Washington, DC: American Psychological Association.

McIlvane, W. J., \& Dube, W. V. (1992). Stimulus control shaping and stimulus control topographies. The Behavior Analyst, 15, 8994. Retrieved from http:/www.ncbi.nlm.nih.gov/pmc/articles/ PMC2733401/pdf/behavan00027-0089.pdf

McIlvane, W. J., \& Dube, W. V. (2003). Stimulus control topography coherence theory: Foundations and extensions. The Behavior Analyst, 26, 195-213. Retrieved from http://www.ncbi.nlm.nih. gov/pmc/articles/PMC2731455/pdf/behavan00006-0017.pdf

McIlvane, W. J., Kledaras, J. B., Munson, L. C., King, K. A. J., de Rose, J. C., \& Stoddard, L. T. (1987). Controlling relations in conditional discrimination and matching by exclusion. Journal of the Experimental Analysis of Behavior, 48, 187-208. doi:10.1901/ jeab.1987.48-187

McIlvane, W. J., Serna, R. W., Dube, W. V., \& Stromer, R. (2000). Stimulus control topography coherence and stimulus equivalence: Reconciling test outcomes with theory. In J. Leslie, \& D. E. Blackman (Eds.), Issues in experimental and applied analysis of human behavior (pp. 85-110). Reno, NV: Context Press.

Ray, B. A. (1969). Selective attention: The effects of combining stimuli which control incompatible behavior. Journal of the Experimental Analysis of Behavior, 12, 539-550. doi:10.1901/jeab.1969.12-539

Reynolds, G. S. (1961). Attention in the pigeon. Journal of the Experimental Analysis of Behavior, 4, 203-208. doi:10.1901/ jeab.1961.4-203

Serna, R. W. (2004). Recent advances in discrimination learning with individuals with developmental disabilities. In L. Williams (Ed.), Developmental disabilities: Etiology, assessment, intervention, and integration. (pp. 81-104). Reno: Context Press.

Schilmoeller, G. L., Schilmoeller, K. J., Etzel, B. C., \& LeBlanc, J. M. (1979). Conditional discrimination after errorless and trial-anderror training. Journal of the Experimental Analysis of Behavior, 31, 405-420. doi:10.1901/jeab.1979.31-405

Sidman, M., \& Stoddard, L. T. (1967). The effectiveness of fading in programming a simultaneous form discrimination for retarded children. Journal of the Experimental Analysis of Behavior, 10, 3-15. doi:10.1901/jeab.1967.10-3

Stromer, R., McIlvane, W. J., Dube, W. V., \& Mackay, H. A. (1993). Assessing control by elements of complex stimuli in delayed matching to sample. Journal of the Experimental Analysis of Behavior, 59, 83-102. doi:10.1901/jeab.1993.59-83

Stromer, R., \& McIlvane, W. J. (1993). Complex stimulus control and equivalence. The Psychological Record, 43, 585-598. Retrieved from EBSCOhost

Terrace, H. S. (1963). Errorless transfer of a discrimination across two continua. Journal of the Experimental Analysis of Behavior, 6, 223-232.

Touchette, P. E., \& Howard, J. S. (1984). Errorless learning: Reinforcements contingencies and stimulus control transfer in delayed prompting. Journal of Applied Behavior Analysis, 17, 175 188. doi:10.1901/jaba.1984.17-175

Wilkinson, K. M., Rosenquist, C., \& McIlvane, W. J. (2009). Exclusion learning and emergent symbolic category formation in individuals with several language impairments and intellectual disabilities. The Psychological Record, 59, 187-206. Retrieved from EBSCOhost

Zygmont, D. M., Lazar, R. M., Dube, W. V., \& McIlvane, W. J. (1992). Teaching arbitrary matching via sample stimulus-control shaping to young children and mentally retarded individuals: A methodological note. Journal of the Experimental Analysis of Behavior, 57, 109-117. doi:10.1901/jeab.1992.57-109. 
